\title{
ReMed: rapport annuel 2020
}

\section{Comité de direction de ReMed}

L'exercice de la médecine implique de s'occuper des autres et de se focaliser sur les besoins des patients, mais il arrive que des médecins traversent eux aussi des périodes difficiles et soient confrontés à des problèmes de santé. Depuis 2007, le réseau ReMed soutient les médecins en situation de crise par diverses offres spécifiques adaptées à leurs besoins.

\section{Nous sommes là pour vous}

Le réseau intervient dans les $72 \mathrm{~h}$ après une première prise de contact par la ou le médecin concerné ou par une personne de son entourage. Le maintien de la santé et de la fonctionnalité du médecin, la garantie de la sécurité des patients et la qualité élevée des soins médicaux sont donc essentiels.

\section{0 prises de contact en 2020}

Le nombre de prises de contact a augmenté de $5 \%$ au cours de l'année écoulée, passant à 170. Cette augmentation constante prouve combien il était urgent qu'un service tel que ReMed voie le jour. Sur ces 170 prises de contact, $64 \%$ des demandes provenaient de Suisse alémanique, $34 \%$ de Suisse romande et $2 \%$ du Tessin. Les appelants étaient pour $60 \%$ des femmes et pour $40 \%$ des hommes. Les problèmes structurels (surcharge au travail et surcharge administrative, entre autres) et les problématiques d'ordre psychique telles que la peur (craintes pour l'avenir, tournants dans la carrière, fermeture et remise de cabinet, crainte de commettre une

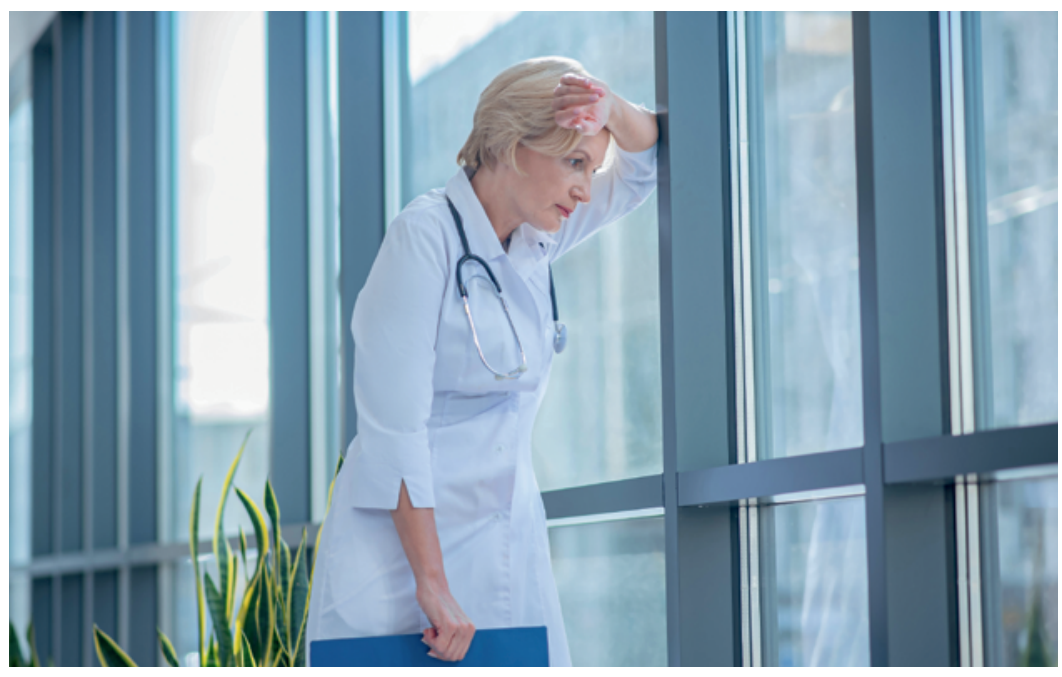

ReMed soutient les médecins qui arrivent au bout de leurs ressources physiques et psychiques. erreur médicale, etc.) ont été les motifs les plus fréquents pour une prise de contact.

\section{Offre étendue durant la crise de coronavirus}

Soucieux de la santé des médecins en cette période de crise liée au coronavirus, ReMed a étendu son offre et engagé plus de personnes pour soutenir les médecins qui arrivent au bout de leurs ressources physiques et psychiques en raison de cette situation particulière. C'est ainsi que les premiers groupes de coaching en ligne ont été mis sur pied en 2020 en Suisse alémanique dans le but de se concentrer sur les solutions et de favoriser l'échange entre collègues sur les défis qu'ils rencontrent. Une petite équipe de médecins a développé trois séances de coaching par visioconférence sous forme de thérapie brève orientée sur les solutions, un outil simple pour connaître les difficultés auxquelles les médecins-assistant-e-s sont actuellement confrontés dans leur activité hospitalière et agir de manière proactive dans le but de prévenir les surcharges.

\section{Groupes de coaching spontanés pour les médecins hospitaliers}

Dans le cadre d'un projet-pilote, ReMed a mis sur pied pour la première fois en 2020 des groupes de coaching spontanés à l'intention des médecins hospitaliers. En respectant strictement les règles d'hygiène et de protection contre le Covid-19, quatre groupes de coaching de deux à trois médecins en formation ont été animés par des membres du comité de direction de ReMed à l'Hôpital universitaire de Zurich. Ces rencontres se poursuivront en 2021.

\section{Evaluation des offres de soutien de ReMed sous l'angle de la qualité des conseils}

La garantie de la qualité et l'extension de l'offre ReMed sont cruciaux pour le comité de direction de ReMed. 


\section{Offres de la FMH relatives à la santé des médecins}

\section{Offre}

ReMed

Coach my Career

Prevention for doctors

Développement professionnel
ReMed

Secrétariat

Case postale 55

CH-3000 Berne

info[at]swiss-remed.ch

\section{Description succincte}

Réseau de soutien pour les médecins en situation de crise. Approche préventive et échange d'expériences. Ligne d'assistance disponible 24 heures sur 24: www.swiss-remed.ch

Offre de mentorat intergénérationnel destiné aux jeunes médecins pour les aider dans la planification de leur formation postgraduée et de leur carrière

Manifestations visant à sensibiliser les médecins aux défis de leur profession pour qu'ils puissent mieux y faire face. Groupe de travail bénéficiant d'un large soutien, chargé de la mise en œuvre de différents projets

Analyse des besoins de la relève médicale et des changements de la démographie médicale. Développement de nouvelles prestations à l'intention des membres de la FMH

Un pas dans ce sens avait déjà été entrepris en 2015 par une évaluation externe (Ettlin, Grunder, Jörg \& Widmer, 2016) qui portait principalement sur la qualité de la structure du programme. Cette étude visait en premier lieu à examiner le budget, l'infrastructure et le personnel au travers d'entretiens, d'évaluations en ligne et d'une analyse des documents de base de ReMed. Le rapport final d'analyse comportait des recommandations qui ont été progressivement mises en œuvre à partir de 2016. De nouvelles personnes ont ensuite été engagées au comité de direction (prise en compte de toutes les régions linguistiques), le processus de répartition des cas a été optimisé et le manuel ReMed et les termes utilisés ont été remaniés. Enfin, des mesures ont été prises pour améliorer la visibilité du réseau.

Soucieux de la santé des médecins en cette période de crise liée au coronavirus, ReMed a étendu son offre.

La nouvelle étude, à laquelle 120 médecins (taux de réponse $42 \%$ ) ayant fait appel à ReMed ont participé entre le $1^{\text {er }}$ novembre 2018 et le 30 avril 2020, vise à évaluer la qualité des consultations. A aucun moment elle n'a porté sur les données personnelles ou les dossiers médicaux. Dans cette enquête, la qualité de la structure a recueilli 97\% d'avis positifs, la qualité de la procédure $90 \%$ et la qualité du résultat $82 \%$.
L'évaluation très positive de la qualité de l'offre par les personnes ayant cherché de l'aide permet de conclure qu'une évaluation externe régulière du travail accompli et l'autocritique sont déterminants pour améliorer la qualité des offres de conseil. Cette démarche permet d'une part de répondre au mieux aux besoins des personnes nécessitant du soutien et, de l'autre, d'approfondir des aspects essentiels de la garantie de la qualité. Cette réflexion sera poursuivie à l'avenir [1].

\section{ReMed - comptes rendus d'expérience et informations}

Le Bulletin des médecins suisses a publié deux nouveaux comptes rendus d'expérience donnant un aperçu des activités de conseil de ReMed. En raison de la pandémie de Covid-19, ReMed n'a pu participer qu'à quelques manifestations et de nombreux événements ont dû être repoussés à 2021. Il a néanmoins été réjouissant de pouvoir proposer des exposés aux étudiants en médecine de l'Université de Zurich ainsi qu'aux médecins-assistant-e-s et chef-fe-s de cliniques de l'Hôpital de Zurich et de l'Hôpital Muri AG.

N'hésitez pas à vous rendre sur notre site internet www.swiss-remed.ch. Vous y trouverez des articles in téressants et des informations importantes sur le réseau ReMed.

Le programme national ReMed, son comité de direction et son réseau d'intervenants seront également là en 2021 pour les médecins en situation de crise. La permanence se tient à leur disposition 24 heures sur 24 et peut être jointe tant par téléphone, au 0800073633 , que par courriel à l'adresse remed[at]hin.ch

\section{Référence}

1 Hadorn L. Travail de Master. Die Gesundheit der Ärztinnen und Ärzte im Fokus. Evaluation der ReMed-Unterstützungstätigkeit mit Fokus auf Beratungsqualität. Anonymisierte Fallauswertung. Arbeit zur Erlangung des Master of Public Health im Rahmen des Weiterbildungsstudiengangs Public Health der Universitäten Basel, Bern und Zürich; 2020 (non publié et non traduit pour l'instant).

Crédits photo

(c) Dmytro Zinkevych | Dreamstime.com (image symbolique) 